\title{
Assessment of Results of Treatment of Paediatric Femoral Diaphyseal Fractures with Titanium Elastic Nails
}

\author{
Devesh Bandil', Megha Gupta Bandil' \\ ${ }^{1}$ Consultant, Department of Orthopedics, ${ }^{2}$ Consultant, Department of OBS and GYN, Dr. Suresh Bandil Memorial Orthopaedic \\ and Maternity Research Center, Gwalior, MP, India
}

Corresponding author: Dr. Megha Gupta Bandil, Consultant, Department of OBS and GYN, Dr. Suresh Bandil Memorial Orthopaedic and Maternity Research Center, Gwalior, MP, India

DOI: 10.21276/ijcmsr.2018.3.2.29

How to cite this article: Devesh Bandil, Megha Gupta Bandil. Assessment of results of treatment of paediatric femoral diaphyseal fractures with titanium elastic nails. International Journal of Contemporary Medicine Surgery and Radiology. 2018;3(2):B121-B123.

\section{A B S T R A C T}

Introduction: Femoral fractures are among the most common fractures of long bones. The management of pediatric femur fractures depends basically on the age group of the child although the age of bone and size of the child also determines the treatment choice. The choice of management may also be determined by surgical experience and local trends in practice. Study aimed to assess the results of treatment of paediatric femoral diaphyseal fractures with titanium elastic nails.

Material and methods: The study was performed Medical institute. A total of 22 patients with closed shaft femur fractures and age ranging between 5-16 years scheduled to undergo treatment with titanium elastic nails were selected for the study. Children with open fractures, outside this age group with concomitant head injury, bilateral femur fracture or associated fracture of either limb whose fixation was done after 7 days were excluded from the study. No control group was used. They were treated by retrograde TENS fixation using two nails of equal diameter for each fracture. Pre-operative evaluation includes full length radiograph of the fractured femur both antero-posterior (A-P) and lateral views.

Results: In the present study, 22 patients with closed shaft femur fractures were treated with titanium elastic nails. The mean age of the patient was 13.23 years. We observed that in regards to fracture location, proximal fracture was seen in 5 patients, midshaft fracture in 14 patients and distal fracture in 3 patients. However, in regards to pattern of location, transverse fracture was seen in 6 patients, oblique fracture in 8 patients, spiral fracture in 5 patients and comminuted fracture in 3 patients.

Conclusion: From this study we can conclude that Intramedullary nailing of the pediatric femoral diaphyseal fractures using titanium elastic nail is an effective surgical modality, with advantages of minimal incisional scar, minimal soft tissue damage, with early union time and excellent union rates, and without affecting shoulder and elbow function.

Keywords: Titanium Elastic Nails, Femoral Diaphyseal Fracture, Orthopedics, Pediatric Femoral Fracture

\section{INTRODUCTION}

Fractures of femur are amongst the most commonly observed fractures of the long bones. ${ }^{1}$ The administration of pediatric femur fractures depend basically on the age group of the child although the age of bone and size of the child may affect the choice of treatment. ${ }^{2}$ The choice of treatment may also be affected by surgical expertise and local trends followed in practice. Non-operative treatment plays a crucial role in some cases still the current practice has swung towards operative fixation as it enables rapid mobilization and shorter hospital stays. The two modalities of internal fixation in fracture shaft of humerus are plate osteosynthesis and intramedullary nailing. ${ }^{3}$ Nails are subjected to smaller bending loads and are less likely to fail due to fatigue. They act as load sharing and stress shielding devices. ${ }^{4}$ Cortical osteopenia that occurs right adjacent to the ends of plates is rarely seen with intramedullary nails; thus, refracture after implant removal is seen less often. ${ }^{5}$ This management method has been the topic of controversy since its beginning because of the damage to medullary blood supply, possibility of fat embolism and a lack of thoughtful of the biomechanical philosophies of intramedullary nail fixation. ${ }^{6}$ Hence, we planned the study to assess the results of treatment of paediatric femoral diaphyseal fractures with titanium elastic nails.

\section{MATERIAL AND METHODS}

The study was performed in the Department of Orthopedics of the Medical institute. The ethical clearance was obtained before the start of study. A total of 22 patients with closed shaft femur fractures and age ranging between 5-16 years scheduled to undergo treatment with titanium elastic nails were selected for the study. Children with open fractures, outside this age group with concomitant head injury, bilateral femur fracture or associated fracture of either limb whose fixation was done after 7 days were excluded from the 


\begin{tabular}{|l|c|}
\hline Parameters & No. of cases \\
\hline Fracture location & 5 \\
Proximal & 14 \\
Midshaft & 3 \\
Distal & \\
\hline Pattern of location & 6 \\
Transverse & 8 \\
Oblique & 5 \\
Spiral & 3 \\
Comminuted & \\
\hline \multicolumn{2}{|c|}{} \\
\hline
\end{tabular}

\begin{tabular}{|l|c|c|c|c|}
\hline $\begin{array}{l}\text { Functional score } \\
\text { (constant score) }\end{array}$ & Loss of range of motion & Radiological union & Subjective symptoms & Number of cases \\
\hline Excellent & $<10 \%$ & Good & No & $18(82 \%)$ \\
\hline Fair & $10-30 \%$ & Good & Mild & $3(14 \%)$ \\
\hline Poor & $>30 \%$ & Good & Moderate & $1(4 \%)$ \\
\hline \multicolumn{2}{|r|}{ Table-2: Assessment of functional score, subjective symptoms and radiological assessment of the cases } \\
\hline
\end{tabular}

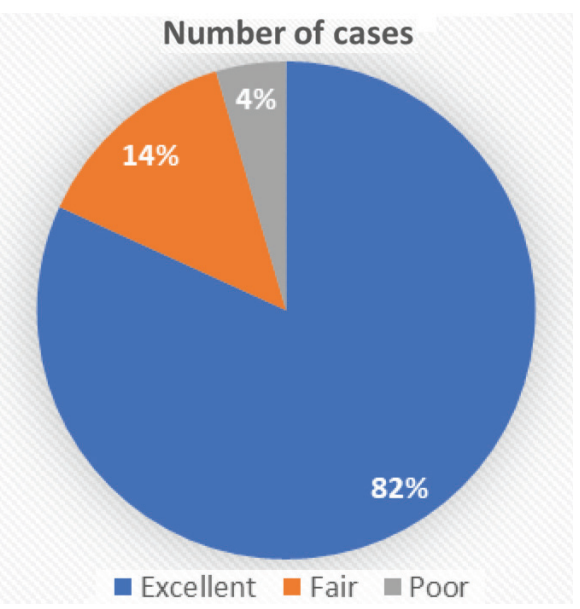

Figure-1: Assessment of functional score, subjective symptoms and radiological assessment of the cases

study. No control group was available in our study. All the subjects were informed about the study and a written consent was obtained.In this study, all the cases were operated within first 8 days of injury. They were treated by retrograde TENS fixation using two nails of equal diameter for each fracture. Pre-operative evaluation includes full length radiograph of the fractured femur both antero-posterior $(\mathrm{A}-\mathrm{P})$ and lateral views (Figure-2). Parameters studied were clinical features of union, radiological features of union, range of motion, mal-alignment and all other complications found during the study.

\section{STATISTICAL ANALYSIS}

The statistical analysis of the data was done using SPSS version 20.0 for windows. Descriptive statistics like mean and percentages were used for the analysis.

\section{RESULTS}

In the present study, 22 patients with with closed shaft femur fractures were treated with titanium elastic nails. The mean age of the patient was 13.23 years. 12 patienst were boys and 10 patients were girls. Table 1 shows the characteristics of femoral shaft fractures. We observed that in regards to

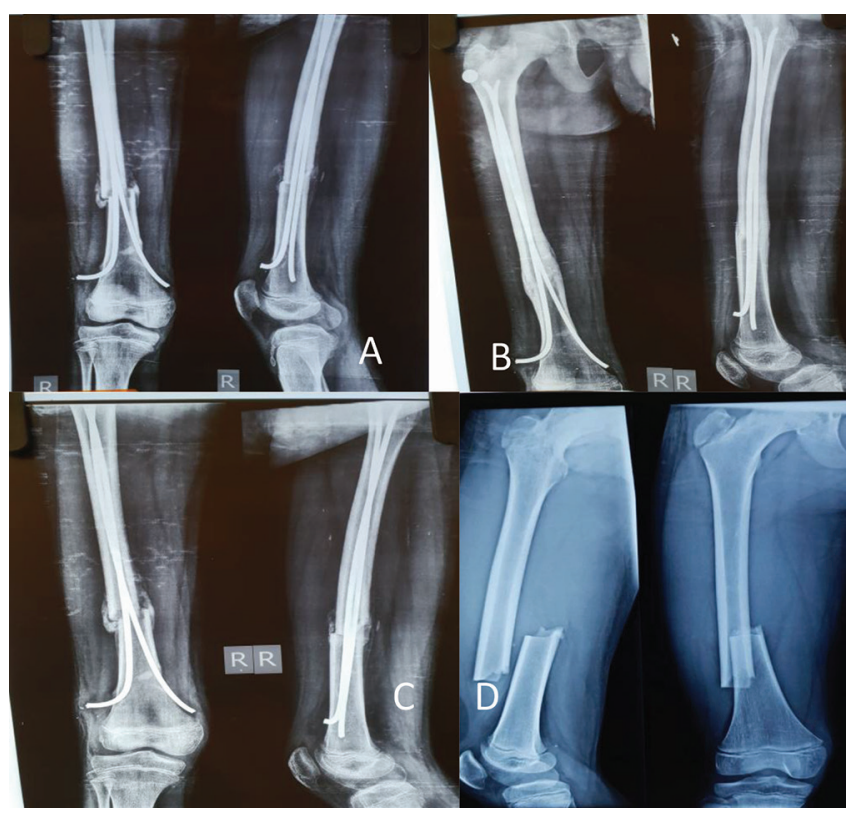

Figure-2: A. Xray showing early callus B.Xray showing well united femoral shaft C. Xrays showing callus at 3 month D. $\mathrm{X}$ ray showing fracture femoral shaft

fracture location, proximal fracture was seen in 5 patients, midshaft fracture in 14 patients and distal fracture in 3 patients. However, in regards to pattern of location, transverse fracture was seen in 6 patients, oblique fracture in 8 patients, spiral fracture in 5 patients and comminuted fracture in 3 patients. Table 2 shows the assessment of functional score, subjective symptoms and radiological assessment of the cases. We observed that excellent functional score is seen in $82 \%$ cases, fair functional score is seen in $14 \%$ cases and poor functional score is seen in $4 \%$ cases.

\section{DISCUSSION}

In the present study we assessed the results of treatment of paediatric femoral diaphyseal fractures with titanium elastic nails. We observed that excellent functional score is seen in $82 \%$ cases, fair functional score was seen in $14 \%$ cases and poor functional score was seen in $4 \%$ cases. But the results were 
statistically non-significant. Comparison of the study results were done with previous studies and results were consistent with previous studies. Papadokostakis G et al compared the efficacy of retrograde nailing in the management of distal femur and shaft fractures. Twenty-four articles were reviewed with a total of 914 patients with a mean age of 48.8 years who had 963 distal and diaphyseal femur fractures. The overall mortality incidence was $5.3 \%$. The rate of infection was $1.1 \%$ and septic arthritis of the knee was seen in $0.18 \%$. Amongst patients with distal femur fractures, the mean union time and union rate were 3.4 months and $96.9 \%$, respectively. The mean range of knee motion was 104.6 degrees. The incidence of pain in knee, malunion and re-operation were $16.5,5.2$ and $17 \%$, respectively. Subjects with femur shaft fractures had a mean union time of 3.2 months, whilst union rate was $94.2 \%$. The mean range of knee motion was 127.6 degrees. The rates of pain in knee, malunion and re-operation were $24.5,7.4$ and $17.7 \%$, respectively. They came to the conclusion that retrograde intramedullary nailing appeared to be reliable management option, chiefly for distal femoral fractures. However, in managing diaphyseal fractures, retrograde intramedullary nailing was associated with high rates of pain and lower rate of fracture union. According to Saikia $\mathrm{K}$ et al management of femoral fractures in pediatric age group is controversial. The study included 26 spediatric subjects with recent femoral diaphyseal fractures that were stabilized using Titanium Elastic Nail. Flynn's scoring criteria was used to evaluate the results. Fischer's exact test was used for statistical analysis. All subjects were followed up for 26 months. Radiological union amongst all cases were seen in a mean duration of 8.7 weeks. Full weight bearing was possible at a mean duration of 8.8 weeks. Mean time of hospital stay was 9.8 days. Excellent results were seen in 13 patients (59.0\%), successful amongst six (27.2\%) and poor amongst three patients (13.6\%). It was concluded that intramedullary fixation titanium elastic nailing is an effective treatment of diaphyseal fractures of the femur in properly selected patients of the 6-16 years age group. ${ }^{7,8}$ Bhuyan $\mathrm{BK}$ et $\mathrm{al}^{9}$ demonstrated the effectiveness of intramedullary fixation of femoral shaft fractures by using titanium elastic nailing system (TENS). Another study conducted in April including 40 pediatric patients between age group of 5-16 years with diaphyseal fractures were managed using retrograde TENS fixation. Classification of fractures was done using Winquest and Hansen scale and compound fractures were classified according to the Gustilo and Anderson's classification. Flynn's criteria were used to describe the result. They found that all fractures were radiologically united callus formation at a mean period of 9 weeks and weight bearing was possible at a time of 9.5 weeks. As per Flynn's criteria, excellent result was observed in $82.5 \%$ patients and satisfactory in $17.5 \%$ subjects. Lengthening of limb was seen in 6 cases, varus mal-alignment was observed in 4 cases and rotational mal-alignment was observed in 3 cases. The study concluded that TENS is a efficient and effective way for the managing of pediatric femoral shaft fractures, as it is minimally invasive, easy to use and has good functional and cosmetic outcome. ${ }^{9}$

\section{CONCLUSION}

From the above study study we conclude that Intramedullary nailing of the pediatric femoral diaphyseal fractures using titanium elastic nail is an effective surgical modality, with advantages of minimal incisional scar, minimal soft tissue damage, with early union time and excellent union rates, and without affecting hip and knee function.

\section{REFERENCES}

1. Garvanos C. Humeral shaft fractures. Rockwood and Green's Fractures in adults. In: InCourt-Brown CM, Heckman JD, McQueen MM, Ricci WMTornetta P III, eds, editors. Philadelphia: Wolters Kluwer Health; 2015. pp. 1287-336. 8th ed.

2. Langer P, Born CT. Intramedullary fixation of humeral shaft fractures. In: InTornetta P III, Williams GR, Ramsey MLHunt TR III, eds, editors. Operative Techniques in Orthopaedic Trauma Surgery. Philadelphia, PA: Lippincott Williams \& Wilkins; 2011.pp. 197-205.

3. Tytherleigh-Strong G, Walls N, McQueen MM. The epidemiology of humeral shaft fractures. J Bone Joint Surg. 1998;80(2):249-53.

4. Bridgman S, Wilson R. Epidemiology of femoral fractures in children in the West Midlands region of England 1991-2001. J Bone Joint Surg Br. 2004;86(8):1152-1157.

5. Harvey AR, Bowyer GW, Clarke NMP. The management of paediatric femoral shaft fractures. Curr Orthop. 2002;16(4):293-299.

6. Schwend RM, Werth C, Johnston A. Femur shaft fractures in toddlers and young children: rarely from child abuse. J Pediatr Orthop. 2000;20(4):475-481.

7. Papadokostakis G, Papakostidis C, Dimitriou R, Giannoudis PV. The role and efficacy of retrograding nailing for the treatment of diaphyseal and distal femoral fractures: a systematic review of the literature. Injury. 2005;36(7):813-22.

8. Saikia K, Bhuyan S, Bhattacharya T, Saikia S. Titanium elastic nailing in femoral diaphyseal fractures of children in 6-16 years of age. Indian J Orthop. 2007;41:381-5.

9. Bhuyan BK, Mohan Singh S. Titanium elastic nailing in pediatric femoral diaphyseal fractures in the age group of 5-16 years - A short term study. J Clin Orthop Trauma. 2014;5(4):203-10.

\section{Source of Support: Nil; Conflict of Interest: None}

Submitted: 05-05-2018; Accepted: 02-06-2018; Published online: 15-06-2018 